\title{
Reviewers for the 2018 IMIA Yearbook of Medical Informatics
}

\author{
Jos Aurts, The Netherlands \\ Mervat Abdelhak, United States \\ François Allaert, France \\ Robert Amland, United States \\ Elske Ammenwerth, Austria \\ Sophia Ananiadou, United Kingdom \\ Jessica Ancker, United States \\ Rzhetsky Andrey, United States \\ Marta Avalos, France \\ Melissa Baysari, Australia \\ Riccardo Bellazzi, Italy \\ Fernan Gonzalez Bernaldo De Quiros, \\ Argentina \\ Jiang Bian, United States \\ Olivier Bodenreider, United States \\ Georgeta Bordea, France \\ Andrew Boyd, United States \\ Benjamin Brown, United Kingdom \\ Alex Bui, United States \\ Anita Burgun, France \\ John Carrino, United States \\ Hubert Cecotti, United States \\ Bagayoko Cheick Oumar, Mali \\ Kevin Bretonnel Cohen, United States \\ Tiago Colicchio, United States \\ Carolina Conejo Gómez, Spain \\ Pascal Coorevits, Belgium \\ Sebastien Cossin, France \\ Kathrin M. Cresswell, United Kingdom \\ Theresa Cullen, United States \\ Dina Demner Fushman, United States \\ Hans Demski, Germany \\ Gayo Diallo, France \\ James Doidge, United Kingdom \\ Alevina Dubovitskaya, Switzerland \\ Martin Dugas, Germany
}

Margo Edmunds, United States

David Eichmann, United States

Frederic Ehrler, Switzerland

Peter Elkin, United States

Peter Embi, United States

Scott Erdley, United States

Sue Feldman, United States

Susan Fenton,United States

Erin Fogarty, United States

Walter Gall, Austria

Jennifer H. Garvin, United States

Carlisle George, United Kingdom

Rosa Gini, Italy

Clément Goehrs, France

Alejandro Rodríguez González, Spain

Graciela Gonzalez-Hernandez, United States

Natalia Grabar, France

Romain Griffier, France

Nicolas Griffon, France

Cyril Grouin, France

Gilles Guezennec, France

Maria Hägglund, Sweden

Angelika Haendel, Germany

Udo Hahn, Germany

Thierry Hamon, France

Sébastien Harispe, France

Paul Harris, United States

Ralf Hofestraedt, Germany

Shannon Houser, United States

Lukas Huber, Austria

Josef Ingenerf, Germany

Trevor Jamieson, Canada

Vianney Jouhet, France

Jayashree Kalpathy-Cramer, United States

Abel Kho, United States
Warren Kibbe, United States

Halil Kilicoglu, United States

Petra Knaup, Germany

Sebastian Köhler, Germany

Dimitrios Kokkinakis, Sweden

Craig Kuziemsky, Canada

Antoine Lamer, France

Paul Landais, France

Francis Lau, Canada

Christoph U. Lehmann, United States

Siaw-Teng Liaw, Australia

Frank Lin, Australia

Gaelle Lissorgues, France

Jack London, United States

Gang Luo, United States

Marci Macdonald, Canada

Nicos Maglaveras, Greece

Romaric Marilly, France

Luis Marco-Ruiz, Norway

Mar Marcos, Spain

Santiago Martinez, Norway

Catalina Martínez Costa, Austria

Deven McGraw, United States

Ginny Meadows, United States

Daniella Meeker, United States

Murad Meghani, United States

Mark Merolli, Australia

Hans Moen, Norway

Pattanasak Mongkolwat, Thailand

Shawn Murphy, United States

Zahra Niazkhani, Iran

Stacy O'Connor, United States

Casey Overby, United States

Bunyamin Ozaydin, United States

Chirag Patel, United States

Carolyn Petersen, United States
David Pieczkiewicz, United States

Andrea Pinna, France

Habibollah Pirnejad, Iran

Maik Plischke, Germany

Morgan Price, Canada

Hans Urich Prokosch, Germany

Bastien Rance, France

Laritza Rodriguez, United States

Wendy Rubinstein, United States

Lipika Samal, United States

Kaija Saranto, Finland

Neil Sarkar, United States

Nikolaus Schulz, United States

Stefan Schulz, Austria

Marco Schweitzer, Austria

Yalini Senathirajah, United States

Liliana Laranio da Silva, Australia

Berglind Smaradottir, Norway

Sunah Song, United States

Felix Sukums, Tanzania

Lina Suliemann, United States

Xavier Tannier, France

Cui Tao, United States

Ye Tian, United States

Annette L. Valenta, United States

Pierre-Yves Vandenbussche, Ireland

Sumithra Velupillai, Sweden

Karin Verspoor, Australia

Amy Wang, United States

Shuang Wang,United States

Thomas Weinhold, Switzerland

Chunhua Weng, United States

Alfred Winter, Germany

Klaus-Hendrik Wolf, Germany

Hua Xu, United States 